\title{
6 Mehrsprachigkeit und DaF-Unterricht
}

\author{
Sabine Grasz
}

Universität Oulu,

Studiengang „Deutsche Sprache und Kultur“

DOI: doi.org/10.31885/9789515150097.6

\section{Rahmendaten}

\begin{tabular}{|c|c|}
\hline Kursname & $\begin{array}{l}\text { Mehrsprachigkeit und DaF-Unterricht - } \\
\text { Teil des Kurses „Sprachdidaktik““ }\end{array}$ \\
\hline $\begin{array}{l}\text { Institution (Universität, } \\
\text { Einrichtung, Fakultät, } \\
\text { Studiengang, Fach) }\end{array}$ & $\begin{array}{l}\text { Universität Oulu, Humanistische Fakultät } \\
\text { Studienprogramm: Sprachen und Kultur } \\
\text { Fach: Deutsche Sprache und Kultur }\end{array}$ \\
\hline Ausrichtung des Kurses & $\begin{array}{l}\text { Einführung in die Fachwissenschaft } \\
\text { Fremdsprachendidaktik }\end{array}$ \\
\hline $\begin{array}{l}\text { Haupt- oder Nebenfach; } \\
\text { BA-, MA-Niveau }\end{array}$ & $\begin{array}{l}\text { Der Kurs ist Teil des Haupt- und Nebenfachstudiums im } \\
\text { Fach „Deutsche Sprache und Kultur“ auf dem BA-Niveau }\end{array}$ \\
\hline $\begin{array}{l}\text { Pflicht-, Wahlpflicht- oder } \\
\text { freiwählbares Fach }\end{array}$ & $\begin{array}{l}\text { Pflichtkurs für Studierende der Studienrichtung Lehramt } \\
\text { Deutsch. Studierende, die nicht Lehramt studieren, können } \\
\text { den Kurs als Wahlpflichtkurs oder freiwählbaren Kurs } \\
\text { absolvieren. }\end{array}$ \\
\hline Leistungspunkte & 5 ECTS für den gesamten Kurs \\
\hline (angestrebte) Gruppengröße & 10-15 Studierende \\
\hline $\begin{array}{l}\text { Kursformat / Umfang } \\
\text { Kontaktunterricht und } \\
\text { Selbststudium }\end{array}$ & $\begin{array}{l}\text { Seminar } \\
\text { Der gesamte Kurs „Sprachdidaktik“ umfasst } 28 \text { Stunden } \\
\text { Kontaktunterricht + } 102 \text { Stunden Selbststudium. Der } \\
\text { Unterricht findet wöchentlich während eines Semesters } \\
\text { (14 Unterrichtswochen) statt. } \\
\text { Die Themeneinheit „Mehrsprachigkeit und DaF- } \\
\text { Unterricht“ umfasst } 6 \text { Stunden Kontaktunterricht und } \\
25 \text { Stunden Selbststudium. }\end{array}$ \\
\hline Unterrichtssprache & Deutsch \\
\hline
\end{tabular}

Grasz, Sabine:

Mehrsprachigkeit und DaF-Unterricht.

In: Kursiša, Anta \& Schlabach, Joachim (Hrsg. | toim. | utg. | eds.) (2020):

PluriंDeutsch - plurilinguale Kurse mit Deutsch. Handreichungen für die Kursentwicklung in der Germanistik und an Sprachenzentren. Helsinki: Universität Helsinki, 137-158.

DOI: doi.org/10.31885/9789515150097 


\section{PlurjoDeutsch}

\begin{tabular}{|c|c|}
\hline Kursname & $\begin{array}{l}\text { Mehrsprachigkeit und DaF-Unterricht- } \\
\text { Teil des Kurses „Sprachdidaktik“ }\end{array}$ \\
\hline $\begin{array}{l}\text { Lehrpersonen } \\
\text { (Sprachlehr- und } \\
\text { Sprachenkompetenzen) }\end{array}$ & $\begin{array}{l}\text { Lehrperson im Fach Deutsch (verantwortliche Person für } \\
\text { Didaktik am Studienfach) } \\
\text { Kompetenzen: Lehrqualifikation, Kenntnis des finnischen } \\
\text { Schulsystems, Kenntnisse in Finnisch, Englisch und } \\
\text { Schwedisch empfehlenswert. }\end{array}$ \\
\hline Zielgruppe & $\begin{array}{l}\text { Haupt- und Nebenfachstudierende im Lehramt Deutsch } \\
\text { des Studienfachs „Deutsche Sprache und Kultur“. } \\
\text { Studierende, die nicht Lehramt studieren, bzw. } \\
\text { Austauschstudierende können bei Interesse auch daran } \\
\text { teilnehmen. }\end{array}$ \\
\hline Sprachen und Niveaus & $\begin{array}{l}\text { Deutsch zumindest B1. } \\
\text { Englisch: keine offizielle Niveauanforderung; das in der } \\
\text { Schule normalerweise erzielte Niveau ist ausreichend für } \\
\text { diesen Kurs. } \\
\text { Finnisch: normalerweise als L1, falls nicht, sind } \\
\text { Grundkenntnisse empfehlenswert, aber nicht unbedingt } \\
\text { erforderlich (z.B. bei Austauschstudierenden). } \\
\text { Weitere Sprachen sind von Vorteil, aber nicht } \\
\text { Voraussetzung. }\end{array}$ \\
\hline $\begin{array}{l}\text { Benötigte Vorkenntnisse, } \\
\text { Fachwissen }\end{array}$ & $\begin{array}{l}\text { Der Kurs gehört zu den Fachstudien. Davor müssen also } \\
\text { schon die Grundstudien in „,Deutsche Sprache und Kultur“ } \\
\text { (25 ECTS) absolviert sein. Keine weiteren } \\
\text { Zugangsvoraussetzungen. }\end{array}$ \\
\hline $\begin{array}{l}\text { Mehrsprachendidaktischer } \\
\text { Ansatz }\end{array}$ & Unterschiedliche Ansätze werden behandelt. \\
\hline Übergeordnetes Lernziel & $\begin{array}{l}\text { Kenntnis von zentralen Inhalten der } \\
\text { Mehrsprachigkeitsdidaktik und Möglichkeiten der } \\
\text { Umsetzung von mehrsprachendidaktischen Ansätzen im } \\
\text { DaF-Unterricht }\end{array}$ \\
\hline
\end{tabular}




\begin{tabular}{|c|c|}
\hline Kursname & $\begin{array}{l}\text { Mehrsprachigkeit und DaF-Unterricht - } \\
\text { Teil des Kurses „Sprachdidaktik“ }\end{array}$ \\
\hline Teillernziele & $\begin{array}{l}\text { Das Konzept Mehrsprachigkeit in seiner historischen } \\
\text { und aktuellen Dimension begreifen und einordnen } \\
\text { können. } \\
\text { Konzepte der Mehrsprachigkeitsdidaktik (v.a. } \\
\text { Tertiärsprachendidaktik und Interkomprehension) } \\
\text { kennen und verstehen. } \\
\text { Die Vorgaben in Bezug auf Mehrsprachigkeit der } \\
\text { finnischen nationalen Lehrpläne für die Grundschule } \\
\text { (Klassen 1-9) und die gymnasiale Oberstufe (POP } \\
\text { 2014 und LOP 2019) (s.u.) kennen und sie in einen } \\
\text { theoretischen Rahmen setzen können. } \\
\text { - Sich mit Unterrichtsmaterialien, die das } \\
\text { mehrsprachige Repertoire der Schüler/innen nutzen, } \\
\text { auseinandersetzen und diese in Hinsicht auf ihre } \\
\text { Einsetzbarkeit im finnischen FS-Unterricht beurteilen } \\
\text { können. } \\
\text {-Fakultativ: Die im Unterricht behandelten Konzepte } \\
\text { und Theorien in die Unterrichtspraxis umsetzen } \\
\text { können.) }\end{array}$ \\
\hline Inhalte, Themen & $\begin{array}{l}\text { Theoretische Auseinandersetzung mit Definitionen von } \\
\text { Mehrsprachigkeit und Konzepten der } \\
\text { Mehrsprachigkeitsdidaktik } \\
\text { Sprachen(lern)biografien } \\
\text { Mehrsprachigkeit in den nationalen Rahmenlehrplänen für } \\
\text { die Grundschule (Klassen 1-9) und die gymnasiale } \\
\text { Oberstufe (POP 2014 und LOP 2019) } \\
\text { Analyse von Unterrichtsmaterialien, die das } \\
\text { mehrsprachige Repertoire der Lernenden berücksichtigen }\end{array}$ \\
\hline Aufgaben & $\begin{array}{l}\text { Lesen, Zusammenfassen und Diskutieren von } \\
\text { wissenschaftlichen Texten } \\
\text { Erstellen einer eigenen Sprachen(lern)biografie } \\
\text { Vorbereiten und Halten einer Kurzpräsentation } \\
\text { Analyse von Unterrichtsmaterialien } \\
\text { (Fakultativ: Vorbereitung, Durchführung und } \\
\text { Nachbereitung einer Unterrichtseinheit) }\end{array}$ \\
\hline Ressourcen & $\begin{array}{l}\text { Handbucheinträge zur Theorie } \\
\text { Perusopetuksen opetussuunnitelman perusteet (POP) } 2014 \\
\text { Lukion opetussuunnitelman perusteet (LOP) } 2019 \\
\text { Gemeinsamer europäischer Referenzrahmen für Sprachen } \\
2001 \\
\text { Gemeinsamer europäischer Referenzrahmen für Sprachen. } \\
\text { Begleitband } 2020 \\
\text { Beispiele aus Lehrwerken }\end{array}$ \\
\hline Lernumgebung & $\begin{array}{l}\text { Seminarraum mit Computer, Projektor } \\
\text { Moodle als Kursplattform }\end{array}$ \\
\hline
\end{tabular}




\section{PlurioDeutsch}

\begin{tabular}{|c|c|}
\hline Kursname & $\begin{array}{l}\text { Mehrsprachigkeit und DaF-Unterricht - } \\
\text { Teil des Kurses „Sprachdidaktik“ }\end{array}$ \\
\hline Bewerten bzw. Prüfen & $\begin{array}{l}\text { Aktive Teilnahme am Unterricht: Kursaktivitäten und } \\
\text { Vorbereitungen/Hausaufgaben (Texte lesen etc.) } \\
\text { Sprachen(lern)biografie } \\
\text { Kurzpräsentation }\end{array}$ \\
\hline $\begin{array}{l}\text { Kursevaluation und - } \\
\text { weiterentwicklung }\end{array}$ & $\begin{array}{l}\text { Kursfeedback durch die Kursteilnehmer/innen } \\
\text { (elektronisches Feedbacksystem) }\end{array}$ \\
\hline $\begin{array}{l}\text { Planung und } \\
\text { Implementierung des Kurses }\end{array}$ & $\begin{array}{l}\text { Testphase: Herbstsemester 2019. Danach Evaluierung } \\
\text { durch Kursleiterin, Kursteilnehmer/innen und } \\
\text { Überarbeitung des Konzepts. } \\
\text { Ab Frühling } 2021 \text { ein fester Bestandteil des Kurses } \\
\text { „Sprachdidaktik“, der einmal im Studienjahr stattfindet. }\end{array}$ \\
\hline $\begin{array}{l}\text { Unterstützende / } \\
\text { behindernde Faktoren }\end{array}$ & $\begin{array}{l}\text { Unterstützend: Mehrsprachigkeit spielt eine zentrale Rolle } \\
\text { in POP und LOP. Passender Kurs für das Thema } \\
\text { Mehrsprachigkeit ist schon im Studienplan vorhanden. } \\
\text { Reform der Studienpläne, die in Zukunft gute } \\
\text { Bedingungen für die Verbindung von Theorie und Praxis } \\
\text { bedeuten, z.B. Möglichkeiten für Schulbesuche sind } \\
\text { gegeben und diese können auch im Studium angerechnet } \\
\text { werden. } \\
\text { Behindernd: Beschränkte zeitliche Ressourcen }\end{array}$ \\
\hline
\end{tabular}

\section{Institutionelle Rahmenbedingungen und Implementierung}

Die Themeneinheit „Mehrsprachigkeit und DaF“ ist ein neu konzipierter Teil des bereits existierenden Kurses „Sprachdidaktik“ am Studienfach „Deutsche Sprache und Kultur“ der Universität Oulu. Das Studienfach gehört zum Studienprogramm „Sprachen und Literatur" der humanistischen Fakultät und bietet neben einer allgemeinen Ausrichtung in Germanistik die Studienrichtungen Lehramt Deutsch und Internationale Wirtschaftskommunikation an.

Im Studienfach „Deutsche Sprache und Kultur“" werden jedes Jahr 12 Studierende im Hauptfach und ca. 15 Studierende im Nebenfach aufgenommen. Zirka ein Drittel der Hauptfach- und zwei Drittel der Nebenfachstudierenden studieren Deutsch im Lehramt, also insgesamt ca. 10-15 Studierende pro Studienjahr. Wie auch an den anderen finnischen Universitäten findet die eigentliche Lehramtsausbildung nicht an den Fächern selbst statt, sondern wird von der erziehungswissenschaftlichen Fakultät gemeinsam mit der Übungsschule der Universität organisiert. Zu dieser 60 ECTS umfassenden Ausbildung gehören allgemeine pädagogische Studien, Fachdidaktikseminare und ein Unterrichtspraktikum. Die Fachdidaktikseminare werden nicht für jedes Fach 
getrennt organisiert, sondern für alle Fremdsprachenfächer (an der Universität Oulu sind das Englisch, Schwedisch, Deutsch) gemeinsam. Für die Lehramtsausbildung muss zu Beginn des Studiums ein Eignungstest bestanden werden.

Zusätzlich zu der 60 ECTS-Lehramtsausbildung bieten die meisten Studienfächer auch eigene Kurse an, die unterschiedliche Aspekte der Didaktik des spezifischen Faches behandeln. Im Studienfach „Deutsche Sprache und Kultur“ der Universität Oulu sind das folgende Kurse:

Sprachdidaktik, 5 ECTS: Pflichtkurs für Lehramtsstudierende in Deutsch im Hauptund Nebenfach im Bachelor-Studium.

Deutsch lernen und lehren, 5 ECTS: Pflichtkurs für Lehramtsstudierende in Deutsch im Hauptfach- und Nebenfach ${ }^{1}$ im Master-Studium.

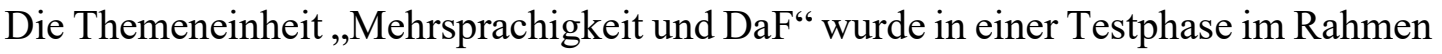
des Kurses „Sprachdidaktik“ im Herbst 2019 erprobt und anschließend evaluiert. Die Implementierung erfolgt im Frühling 2021. Es ist geplant, den Kurs einmal im Studienjahr anzubieten.

\section{Didaktisch-theoretischer Bezugsrahmen}

Bei der Planung und Durchführung des Kurses „Sprachdidaktik“ sind vielfältige didaktische und theoretische Rahmenbedingungen zu berücksichtigen. So dient der Kurs einerseits als eine Einführung in das Thema DaF-Didaktik, in dessen Rahmen sich die Studierenden theoretisch/wissenschaftlich mit aktuellen Themen und Theorien auseinandersetzen sollen. Andererseits soll es aber im Rahmen der Ausbildung angehender Fremdsprachenlehrer/innen nicht bei der rein theoretischen Beschäftigung mit dem Thema bleiben, sondern auch um die konkrete Umsetzung in die Unterrichtspraxis gehen. Außerdem ist zu berücksichtigen, dass bei allen Kursen einer Fremdsprachenphilologie immer auch die Erweiterung und Festigung der Sprachkompetenz der Studierenden (z.B. in verschiedenen Fachsprachen) ein integraler Bestandteil ist, auch bei solchen Kursen, die keine sprachpraktischen Übungen im engeren Sinne sind.

Alle diese Aspekte werden auch im hier vorgestellten Kurs bzw. in der Themeneinheit berücksichtigt, was eine Vielschichtigkeit der Kursinhalte und Ziele mit sich bringt:

- Kennenlernen und Auseinandersetzung mit Theorien und Forschungsarbeiten zur Mehrsprachigkeitsdidaktik und zu verwandten Konzepten

- Auseinandersetzung damit, wie diese Theorien im konkreten institutionellen Kontext umgesetzt werden, also z.B. in den nationalen Curricula und in Lehrwerken

- Umsetzung (in eingeschränktem Rahmen) der von der Theorie und dem institutionellen Rahmen vorgegebenen Konzepte in die Unterrichtspraxis

\footnotetext{
${ }^{1}$ Nebenfachstudierende absolvieren aber nur selten Masterstudienkurse im Nebenfach.
} 


\section{PlurioDeutsch}

- Reflexion des eigenen Sprachlernprozesses und Erweiterung des Sprachwissens und -könnens in Hinblick auf mehrsprachige Aktivitäten

Die Auswahl der Themeneinheit „Mehrsprachigkeit und DaF“ sowie der beiden anderen Themeneinheiten („Früher Fremdsprachenunterricht/Sprachduschen“ und „Digitale Medien im DaF-Unterricht") im Kurs „Sprachdidaktik“ beruht auf den Bedingungen und Trends des Fremdsprachenunterrichts im Allgemeinen und des DaF-Unterrichts an finnischen Schulen im Besonderen. So spielt Mehrsprachigkeitsdidaktik und die Berücksichtigung des gesamten sprachlichen Repertoires der Lernenden als Ressource im Fremdsprachenunterricht schon länger eine zentrale Rolle in der Fremdsprachendidaktik. Diese spiegelt sich nun auch in den aktuellen Rahmenlehrplänen in Finnland wider (POP 2014 und LOP 2019). Gerade in Hinblick auf den Deutschunterricht in Finnland sind plurilinguale Ansätze zentral, da Deutsch fast immer neben und nach anderen Sprachen (vor allem Englisch und Schwedisch) gelernt wird. Ähnlich verhält es sich in Hinblick auf die Sprachenverwendung: Situationen, in denen Fremdsprachen verwendet werden, sind sehr häufig plurilingual - z.B. bei der Verwendung von Deutsch im Arbeitsleben, aber auch auf Reisen und im privaten Bereich. Deshalb ist es notwendig, nicht nur eine Sensibilisierung für Mehrsprachigkeit zu fördern, sondern auch die Studierenden konkret auf plurilinguale Situationen vorzubereiten bzw. im Falle von zukünftigen Fremdsprachenlehrer/innen, sie dazu zu befähigen, ihre Schüler/innen darauf vorzubereiten. Das erscheint auch als besonders wichtig und notwendig, da im Zuge einer Umfrage unter Lehramtsstudierenden (Grasz 2020) herausgefunden wurde, dass diese selbst nur wenig Erfahrung mit Mehrsprachigkeitsdidaktik aus ihrer eigenen Schulzeit haben.

\section{Beschreibung der Kursstruktur und der Lerneinheiten}

Der Kurs „Sprachdidaktik“ umfasst insgesamt 28 Stunden Kontaktunterricht und 102 Stunden Selbststudium. Der Unterricht findet wöchentlich während eines Semesters (14 Unterrichtswochen) statt. Er gehört zu den Fachstudien, was bedeutet, dass die Grundstudien (25 ECTS) vor Besuch des Kurses schon absolviert sein müssen. Normalerweise wird der Kurs im zweiten Studienjahr besucht. Wie schon oben in der Übersicht erwähnt, ist der Kurs ein Pflichtkurs für Lehramtsstudierende in Deutsch im Haupt- und Nebenfach im Bachelor-Studium. Er kann auch von Studierenden, die nicht Lehramt studieren, als wahlfreier Kurs gewählt werden. Die übliche Teilnehmerzahl ist 10-15 Studierende und der Kurs wird einmal pro Studienjahr angeboten.

In der ersten Hälfte des Kurses wird ein Überblick über Prinzipien und Methoden des Fremdsprachenunterrichts (mit Schwerpunkt DaF) gegeben (10 Stunden Kontaktunterricht +12 Stunden Selbststudium), im zweiten Teil werden drei Themen behandelt: Früher Deutschunterricht/Sprachduschen, digitale Medien im DaF-Unterricht und Mehrsprachigkeit und DaF. Ab dem Studienjahr 2020/2021 ist möglich, parallel zu 
dem Kurs freiwillig einen Didaktik-Workshop zu absolvieren. In dessen Rahmen sollen die im Kurs theoretisch behandelten Themen auch praktisch umgesetzt werden. Konkret wird das bedeuten, dass die Studierenden selbst Unterrichtseinheiten zu einem der drei Themen vorbereiten und im Rahmen von Schulbesuchen umsetzen.

Für jedes Thema sind 6 Stunden Kontaktunterricht und 30 Stunden Selbststudium reserviert. Der Stundenumfang ist sehr knapp bemessen und kann - falls möglich - erweitert werden. Die Themeneinheit „Mehrsprachigkeit und DaF“, die im Rahmen des PlurioDeutsch-Projektes entwickelt wurde, ist in drei thematische Lerneinheiten geteilt, die im Folgenden genauer beschrieben werden: Einführung in das Thema Mehrsprachigkeit; Mehrsprachigkeit als Ressource beim Lernen und Verwenden von Sprachen; Mehrsprachigkeit in der Unterrichtspraxis: die finnischen nationalen Rahmenlehrpläne, Unterrichtsmaterialien und plurilinguale Aktivitäten.

\section{Lerneinheit 1:}

\section{Einführung in das Thema Mehrsprachigkeit}

\section{Ziel der Lerneinheit}

Die Studierenden sollen durch die Auseinandersetzung mit unterschiedlichen Definitionen von Mehrsprachigkeit in ihrer historischen Entwicklung die Komplexität und Vielschichtigkeit des Begriffs verstehen. Sie sollen die Definitionen mit ihren subjektiven Theorien darüber und Erfahrungen damit, was Mehrsprachigkeit bedeutet, spiegeln. Sie sollen ihr eigenes mehrsprachiges Repertoire sowie Sprachlern- und Sprachverwendungserfahrungen, die sie in formalen und informellen Kontexten gesammelt haben, reflektieren und sich dadurch der Vielfalt von mehrsprachigen Biografien bewusst werden.

\section{Aufbau der Lerneinheit}

Die Lerneinheit besteht aus den folgenden zwei Teilen:

1) Exemplarische Definitionen zur Mehrsprachigkeit und ihre historische Entwicklung

2) Das eigene mehrsprachige Repertoire und vielfältige Sprachen(lern)biografien

1) Exemplarische Definitionen zur Mehrsprachigkeit und ihre historische Entwicklung

\section{Benötigte Ressourcen/Quellen}

Ein Arbeitsblatt mit Definitionen (bzw. Beschreibungen) von Mehrsprachigkeit aus verschiedenen Zeiten. Welche Definitionen und aus welchen Zeitperioden diese gewählt werden, kann je nach Zielgruppe entschieden werden. Für den betreffenden Kurs 


\section{PlurioDeutsch}

werden Definitionen aus den letzten 100 Jahren verwendet (siehe Anhang 1), die deutlich die Entwicklung des Verständnisses von Mehrsprachigkeit dokumentieren. Eine zentrale Beschreibung stammt aus dem Gemeinsamen europäischen Referenzrahmen für Sprachen (2001), weil dieser einen großen Einfluss auf die finnischen Lehrpläne für den Fremdsprachenunterricht hat.

\section{Mögliche Aufgabenstellung}

Da der Kontaktunterricht im betreffenden Kurs sehr begrenzt ist, bekommen die Studierenden das Arbeitsblatt mit den Definitionen und folgendem Auftrag eine Woche vor der Stunde:

- Lies die verschiedenen Textausschnitte und versuche die zentralen Kriterien für die Definitionen von Mehrsprachigkeit herauszuarbeiten.

- Welche Veränderungen im Verständnis von Mehrsprachigkeit lassen sich in den Definitionen erkennen?

- Welche der Definitionen entspricht am besten deinem eigenen Verständnis von Mehrsprachigkeit?

$\mathrm{Zu}$ Beginn der Unterrichtsstunde werden die Studierenden in Kleingruppen geteilt, in denen sie die vorbereiteten Aufgaben vergleichen und danach diskutieren.

Wenn mehr Kontaktunterricht zur Verfügung steht, können die Texte auch in der Stunde gelesen werden und die Fragen gemeinsam in Partner- und Kleingruppenarbeit beantwortet und diskutiert werden.

Die Definitionen sind zum Teil komplex und es ist anzunehmen, dass das Verständnis von Sprache(n) als (zumindest im Gebrauch) nicht streng trennbare Einheiten, das zum Beispiel den Definitionen der letzten Jahre (Busch 2017) zugrunde liegt, für viele Studierende neu ist und Fragen aufwerfen kann. Deshalb ist empfehlenswert, die Ergebnisse und Diskussionen aus der Kleingruppenarbeit im Plenum zusammenfassend zu präsentieren. Dabei können mögliche offene Fragen gemeinsam geklärt werden.

\section{Begründung der Vorgehensweise}

Durch die Auseinandersetzung mit verschiedenen Definitionen zu Mehrsprachigkeit können die Studierenden einen guten Überblick darüber bekommen, was unter Mehrsprachigkeit verstanden wird. Die Verwendung von Definitionen aus unterschiedlichen Zeiten soll verdeutlichen, dass das Verständnis von individuellen und gesellschaftlichen Konzepten - wie Mehrsprachigkeit es ist - immer Veränderungen unterliegt. Diese Veränderungen beruhen auf neuen wissenschaftlichen Erkenntnissen sowie auf Änderungen gesellschaftlicher Realitäten. Die Definitionen verdeutlichen außerdem sehr gut die zunehmende sprachliche Diversität, wie sie auch in Finnland zu erkennen ist und auf die gesellschaftlich wichtige Institutionen, wie Schulen, reagieren müssen. 


\section{2) Das eigene Sprachenrepertoire und vielfältige Sprachen(lern)biografien}

\section{Benötigte Ressourcen/Quellen}

Material (Papier und Stift oder Laptop) für Notizen.

\section{Mögliche Aufgabenstellung}

Bei dieser Aufgabe sollen die Studierenden ihre eigene Mehrsprachigkeit reflektieren und sich der Vielfalt von Sprachen(lern)biografien bewusst werden. Sie sollen auch erste Überlegungen dazu anstellen, ob ihnen ihr mehrsprachiges Repertoire als Ressource beim Lernen und Verwenden von Sprachen dient.

Das geschieht, indem sie zuerst alle in Einzelarbeit ihr eigenes mehrsprachiges Repertoire und die eigenen Sprachenlern- und Sprachenverwendungserfahrungen reflektieren und dabei Notizen machen.

Folgende (oder ähnliche) Fragen können vorgegeben werden:

- Welche Sprachen habe ich wann, wo und wie lange gelernt?

- Wie gut beherrsche ich die Sprachen (verschiedene Fertigkeiten berücksichtigen)?

- Welche Sprachen verwende ich derzeit regelmäßig? In welchen Kontexten?

- Welche Sprachen habe ich früher verwendet? In welchen Kontexten?

- Welche Rolle spielen die verschiedenen Sprachen bei meinem Sprachenlernen und in der Kommunikation in Fremdsprachen? Unterstützen sie sich gegenseitig? Verwende ich sie als Ressource? Stören sie?

Nach der Reflexionsphase folgt ein Austausch und Vergleich der Erfahrungen in Kleingruppen. In diesem Zusammenhang sollen die Studierenden auch noch einmal auf die in der ersten Aufgabe behandelten Definitionen von Mehrsprachigkeit eingehen und diese mit den verschiedenen Formen von Mehrsprachigkeit, die in der Kleingruppe vertreten sind, vergleichen.

Auf der Basis der im Unterricht gemachten Notizen und der Diskussionen sollen die Studierenden zuhause ihre eigene Sprachen(lern)biografie verfassen, die der Kursleiterin abgegeben wird.

\section{Begründung der Vorgehensweise}

Das Verfassen einer Sprachen(lern)biografie soll die Studierenden zu einer Reflexion ihrer eigenen Mehrsprachigkeit und ihrer Sprachenlern- und Sprachenverwendungserfahrungen anregen. Durch die Diskussion mit den Kommiliton/innen wird angestrebt, dass sie sich der Vielfalt von mehrsprachigen Biografien bewusst werden. Dabei soll es - auch auf der Grundlage der zu Beginn der Lerneinheit kennengelernten Definitionen von Mehrsprachigkeit - zu einer kritischen Auseinandersetzung mit eigenen Auffassungen von und Einstellungen gegenüber Mehrsprachigkeit und vielleicht auch zu einer Revision überkommener Vorstellungen kommen. Die Studierenden sollen sich 
dabei auch erste Gedanken darüber machen, wie die eigene Mehrsprachigkeit als Ressource beim Lernen und Verwenden von Sprachen genutzt werden kann, was dann in der nächsten Lerneinheit genauer behandelt wird.

Gleichzeitig lernen die Studierenden durch das Verfassen einer eigenen Sprachen(lern)biografie eine Aktivität kennen, die sie in Zukunft als Fremdsprachenlehrende selbst im Unterricht einsetzen können.

\section{Bewertung}

Die Aktivität der Studierenden in den Gruppendiskussionen und bei den Diskussionen im Plenum werden für die ganze Lerneinheit, aber nicht für jede einzelne Aktivität bewertet ${ }^{2}$. Dabei wird berücksichtigt, ob die Studierenden die Texte zuhause gelesen haben, ob sie die Unterschiede zwischen den Definitionen herausarbeiten können und ob sie in der Diskussion versuchen, die Theorie mit ihrem eigenen Verständnis von Mehrsprachigkeit in Verbindung zu setzen. Auch die Sprach(lern)biografien fließen in die Benotung der Aktivität ein. Diese müssen eine Tiefe der Reflexion der eigenen Erfahrungen und eine gründliche Auseinandersetzung mit dem Thema spiegeln, um akzeptiert zu werden.

\section{Lerneinheit 2:}

\section{Mehrsprachigkeit als Ressource beim Lernen und Verwenden von Sprachen}

\section{Ziel der Lerneinheit}

In dieser Lerneinheit sollen die Studierenden verstehen, wie das mehrsprachige Repertoire eines Individuums als Ressource einerseits beim Lernen von Fremdsprachen und anderseits beim Verwenden von Sprachen dienen kann. Sie kennen zentrale Theorien und Konzepte zum multiplen Sprachenlernen und zur Mehrsprachigkeitsdidaktik sowie plurilinguale Kompetenzen.

\section{Aufbau der Lerneinheit}

Diese Lerneinheit besteht aus den folgenden zwei Teilen:

1) Theorien und Konzepte zur Mehrsprachigkeitsdidaktik

2) Plurilinguale Kompetenzen

\footnotetext{
${ }^{2}$ Aktivität im Kurs macht $20 \%$ der Kursnote aus und wird mithilfe einer 5-teiligen Skala (5 = ausgezeichnet, 1 = ausreichend) bewertet.
} 


\section{1) Theorien und Konzepte zur Mehrsprachigkeitsdidaktik}

\section{Benötigte Ressourcen/Quellen}

Handbuchtexte; die Studierenden benötigen Laptops (zumindest einen pro Kleingruppe) für die Aufgaben auf der Kursplattform Moodle.

In diesem Kurs werden die unten aufgelisteten Texte verwendet. Es handelt sich vorrangig um Texte aus Handbüchern, da diese vom Umfang her passend sind, einen guten Überblick über die Themen liefern und auf weiterführende Literatur hinweisen. Selbstverständlich können auch andere Texte verwendet werden und es empfiehlt sich, die Literaturliste in regelmäßigen Abständen zu aktualisieren.

- Hans-Jürgen Krumm / Hans H. Reich (2016): Ansätze zum Mehrsprachigkeitsunterricht

- Franz-Joseph Meißner (2016): Interkomprehension

- Nicole Marx (2016): Lernen von zweiten und weiteren Fremdsprachen im Sekundarschulalter

Alternativ (oder ergänzend) bieten sich auch folgende Texte an:

- Nicole Marx / Britta Hufeisen (2010): Mehrsprachigkeitskonzepte

- Wolfgang Hallet / Frank G. Königs (2019, 3. Aufl.): Mehrsprachigkeit und vernetzendes Sprachlernen

\section{Mögliche Aufgabenstellung}

Die drei ausgewählten Handbuchartikel werden den Studierenden zugeteilt. Je nach Gruppengröße sollen 3-4 Studierende denselben Text bis zur nächsten Stunde lesen. Außerdem müssen eine Kurzpräsentation (max. 10 Minuten) und ein Handout mit den wichtigsten Inhalten vorbereitet werden. Das Handout kann auch die Form einer Mindmap haben.

In der Stunde werden die Studierenden in Dreiergruppen (pro Text eine Person) geteilt, in denen sie sich gegenseitig als Experten die gelesenen Texte unter Verwendung der Handouts präsentieren. Danach sollen die einzelnen Ansätze verglichen werden und dabei ein Arbeitsblatt, das auf der Kursplattform zugänglich und direkt bearbeitbar ist, gemeinsam ausgefüllt werden. Im Arbeitsblatt sollen

1) die Hauptmerkmale der einzelnen Ansätze,

2) Gemeinsamkeiten zwischen den Ansätzen und

3) Unterschiede zwischen den Ansätzen festgehalten werden.

Nach den Kurzpräsentationen und Kleingruppendiskussionen folgt auf der Basis der Arbeitsblätter ein zusammenfassender Überblick über die Ansätze der Mehrsprachigkeitsdidaktik durch die Kursleiterin mit anschließender Diskussion bzw. Gelegenheit für Fragen. 


\section{PlurioDeutsch}

\section{Begründung der Vorgehensweise}

Das Lesen der Handbuchtexte und die Vorbereitung der Kurzpräsentationen sollen die Studierenden zu einer aktiven Auseinandersetzung mit dem Thema anregen. Durch die begrenzt zur Verfügung stehende Zeit ist es nicht möglich, längere Texte bzw. Bücher zum Thema Mehrsprachigkeit im Kurs lesen zu lassen. Die kurzen Handbuchartikel können aber einen guten Überblick über das Thema liefern und sind aus diesem Grund für eine Einführungsveranstaltung passend. Gleichzeitig mit dem theoretischen Einblick in die Thematik werden Leseverständnis (von wissenschaftlichen Fachtexten) und Präsentationsfähigkeit geübt. Die gemeinsame Erarbeitung der Hauptmerkmale und der möglichen Gemeinsamkeiten bzw. Unterschiede zwischen den Ansätzen soll zu einem tieferen Verständnis der einzelnen Ansätze beitragen.

\section{2) Plurilinguale Kompetenz}

\section{Benötigte Ressourcen/Quellen}

Kurzvorlesung durch Kursleiterin (kann als Video auf der Kursplattform Moodle zur Verfügung gestellt werden)

Folgende Ausschnitte aus Gemeinsamer europäischer Referenzrahmen für Sprachen: lernen, lehren, beurteilen. Begleitband (GeR-Begleitband 2020) ${ }^{3}$ :

„Mediation” (S. 112-143), davon besonders die Deskriptoren „Spezifische Informationen weitergeben” (S. 116-117), „Daten (in Grafiken, Diagrammen, Schaubildern usw.) mündlich oder in Gebärdensprache erklären ” (S. 118-119), „Mündliche Verarbeitung von Texten (oder in Gebärdensprache)” (S. 120-121), „Einen geschriebenen Text mündlich oder in Gebärdensprache übersetzen” (S. 124-125), „Als Mittler agieren in informellen Situationen (unter Freunden und Kollegen/Kolleginnen)“ (S. 137).

„Plurilinguale und plurikulturelle Kompetenz” (S 144-151), davon besonders die Deskriptoren „Plurilinguales Verstehen” (S. 148-149) und „Auf einem plurilingualen Repertoire aufbauen“(S. 150-151).

\section{Mögliche Aufgabenstellung}

Für diesen Teil der Lerneinheit bietet sich eine Kurzvorlesung an, in der ein Überblick über den Begriff „Plurilinguale Kompetenz“ im GeR-Begleitband (2020) gegeben wird. Hierbei liegt also der Fokus mehr auf der Nutzung des plurilingualen Repertoires als Ressource in Sprachverwendungssituationen, insbesondere auf den Aktivitäten Mediation, plurilinguale Texterschließung und Sprachenwechsel.

\footnotetext{
${ }^{3}$ In der Pilotphase 2019 wurde die im Internet frei zugängliche englische Ausgabe Common European Framework of Reference for Languages: Learning, Teaching, Assessment. Companion volume with new descriptors (2018) verwendet. Bei der Überarbeitung der vorliegenden Kursbeschreibung wurden die betreffenden Textstellen aus der im Jahr 2020 erschienenen deutschen Ausgabe angegeben.
} 
Alternativ kann die Kurzvorlesung im Sinne der flipped classroom-Methode auch auf der Kursplattform Moodle bereitgestellt werden. Die Studierenden sehen sich diese Vorlesung vorab an und in der Stunde werden nur kurz eventuelle Fragen der Studierenden zum Thema geklärt.

Wenn noch Zeit bleibt, kann die Einheit mit einer kurzen Reflexionsphase in Kleingruppen abgeschlossen werden, bei der folgende Fragen dem Erfahrungsaustausch dienen können:

- Habt ihr die in der Vorlesung und in den Deskriptoren beschriebenen plurilingualen Aktivitäten (Mediation, plurilinguale Texterschließung, Sprachenwechsel) schon verwendet? Wenn ja, gebt Beispiele!

- Wie würdet ihr eure eigene plurilinguale Kompetenz (also die Fähigkeit, mithilfe plurilingualer Strategien bestimmte Kommunikationssituationen zu meistern) einschätzen?

- Wie könnte plurilinguale Kompetenz im Sprachunterricht trainiert werden?

Falls in der Stunde keine Zeit dafür ist, kann diese Reflexionsaktivität auch als Hausaufgabe gegeben werden. Diese Reflexionen können dann zum Beispiel in ein Diskussionsforum auf der Kursplattform Moodle gestellt und dort diskutiert werden.

\section{Begründung der Vorgehensweise}

Die Entscheidung, das Thema „Plurilinguale Kompetenz“ in Form von einer Kurzvorlesung zu behandeln, beruht hauptsächlich auf pragmatischen Gründen. Durch die Vorlesung kann das Thema in komprimierter Form präsentiert werden. Die Studierenden werden auch etwas entlastet, da die Einführung es ihnen erleichtern soll, die Deskriptoren schnell zu erfassen. Das erscheint auch deswegen als sinnvoll, da die Lesbarkeit der Deskriptoren nicht optimal ist und es keine scharfe Trennung zwischen interlingualen (=plurilingualen) und intralingualen Strategien gibt, was für Unklarheiten sorgen kann.

\section{Bewertung}

Die Kurzpräsentationen und die Handouts (müssen in der Kursplattform Moodle hochgeladen werden) werden benotet. Dabei wird bewertet, wie gut und verständlich die in den Handbuchtexten dargestellten Theorien und Konzepte wiedergegeben werden. Außerdem wird auch hier die Aktivität in den Diskussionen (in der Stunde und/oder im Diskussionsforum der Kursplattform) in Hinblick auf die Tiefe in der Auseinandersetzung mit den Fragen zur plurilingualen Kompetenz und den damit verbundenen Deskriptoren bewertet. 


\section{PlurioDeutsch}

\section{Lerneinheit 3:}

\section{Mehrsprachigkeit in der Unterrichtspraxis: die finnischen nationalen Rahmenlehrpläne, Unterrichtsmaterialien und plurilinguale Aktivitäten}

\section{Ziel der Lerneinheit}

Die Studierenden setzen sich mit der Rolle von Mehrsprachigkeit in den aktuellen finnischen nationalen Rahmenlehrplänen (POP 2014 und LOP 2019) auseinander und sind fähig, diese in einem weiteren theoretischen Rahmen zu verorten. Sie sollen auch die Veränderungen, die sich seit ihrer eigenen Schulzeit vollzogen haben, reflektieren.

Die Studierenden sollen unterschiedliche Unterrichtsmaterialien und plurilinguale Aktivitäten kennen und die Einsetzbarkeit dieser Aktivitäten und Materialien beurteilen können.

\section{Aufbau der Lerneinheit}

Diese Lerneinheit besteht aus den folgenden zwei Teilen:

1) Mehrsprachigkeit in den finnischen nationalen Rahmenlehrplänen

2) Unterrichtsmaterialien.

\section{1) Mehrsprachigkeit in den finnischen nationalen Rahmenlehrplänen}

\section{Benötigte Ressourcen/Quellen}

Ein Handout mit Ausschnitten aus den aktuellen finnischen Rahmenlehrplänen (POP 2014 und LOP 2019), in denen auf Mehrsprachigkeit eingegangen wird. Anhang 2 zeigt, wie das Handout aussehen könnte. Die Textauszüge dafür wurden aus der englischsprachigen Version des nationalen Rahmenlehrplans für die Gemeinschaftsschule (Klassen 1-9) (POP 2014) genommen. Im Kurs wird normalerweise die finnische Originalversion verwendet, falls aber nicht Finnisch sprechende (Austausch-)Studierende teilnehmen, kann auch die englische Version herangezogen werden. Entsprechende Stellen können auch aus dem Lehrplan für die gymnasiale Oberstufe (LOP 2019) verwendet werden. Das Handout kann ausgeteilt werden, in diesem Kurs ist es auf der Kursplattform Moodle zugänglich.

\section{Mögliche Aufgabenstellung}

Die Studierenden sollen die Textausschnitte aus dem Lehrplan zuhause lesen und sich stichwortartige Notizen zu den folgenden Fragen machen. (Falls im Kurs mehr Zeit zur Verfügung steht, kann das auch direkt im Unterricht geschehen.) 
- Sind euch Aspekte, wie sie in den aktuellen Lehrplänen beschrieben werden, aus eurer eigenen Schulzeit bekannt?

- War Mehrsprachigkeit in eurer Schulzeit ein Thema allgemein bzw. im Sprachenunterricht?

- Wenn ja, welche konkreten Beispiele dafür könnt ihr geben?

- Welchen Bezug zwischen der im Lehrplan beschriebenen Rolle von Mehrsprachigkeit und den im Kurs bisher behandelten Theorien und Konzepten zur Mehrsprachigkeit könnt ihr erkennen?

- Wie beurteilt ihr die in den Lehrplänen behandelten Aspekte (auch in Hinblick auf eigene Lern- und vielleicht auch Unterrichtserfahrungen)?

In der Stunde werden die Studierenden in Gruppen von drei bis maximal vier Personen geteilt, in denen sie ihre Erfahrungen und Meinungen vergleichen. Nach den Kleingruppengesprächen werden die einzelnen Fragen im Plenum kurz gemeinsam durchgegangen.

\section{Begründung der Vorgehensweise}

Es kann davon ausgegangen werden, dass Mehrsprachigkeit zu der Zeit, als die Studierenden selbst die Schule besucht haben, eine kleine Rolle spielte, da diese in den früheren Rahmenlehrplänen nur wenig behandelt wurde. Deshalb erscheint es als wichtig, die Neuerungen in den Lehrplänen ausführlich zu reflektieren und in einen theoretischen Zusammenhang zu stellen. Da die Studierenden in Zukunft als Fremdsprachenlehrer/innen arbeiten werden, ist auch die Auseinandersetzung mit den Lehrplänen und den darin enthaltenen Vorgaben wichtig.

\section{2) Unterrichtsmaterialien und plurilinguale Aktivitäten}

\section{Benötigte Ressourcen/Quellen}

Stationentische mit Kopien von Aufgaben/Aktivitäten, die entweder auf das mehrsprachige Repertoire der Lernenden aufbauen oder die auch plurilingual (im Sinne einer parallelen Verwendung von mehreren Sprachen) ausgerichtet sind. Die Anzahl der Tische sollte je nach Kursgröße so berechnet werden, dass nicht mehr als zwei bis maximal vier Studierende gleichzeitig an einem Tisch sind.

Für diesen Kurs werden hauptsächlich Aufgaben aus der Materialiensammlung „Mehr als Deutsch! Mehrsprachiges Lehrmaterial für den Deutschunterricht“ (hrsg. v. Kursiša und Richter-Vapaatalo 2018) verwendet. Das Material ist für diesen Kurs besonders gut geeignet, weil es sich direkt auf den finnischen Schulkontext bezieht und neben der Erstsprache Finnisch die üblicherweise gelernten Fremdsprachen Schwedisch und Englisch berücksichtigt. Außerdem gibt es Hinweise auf die Niveaustufen und die Klassen, in denen das Material eingesetzt werden kann. Ergänzt wird das Material mit Aufgaben, in denen mehrere Sprachen parallel in einer Situation verwendet werden (bspw. aus Beiträgen im vorliegenden Band), da diese Aktivitäten in „Mehr als Deutsch!“ nicht enthalten sind. 


\section{PlurjoDeutsch}

\section{Mögliche Aufgabenstellung}

Die Studierenden gehen von Stationentisch zu Stationentisch, dabei probieren sie zuerst die Aufgabe selbst aus und diskutieren danach die Aufgabe mit den anderen Studierenden am Tisch. Folgende Fragen sind vorgegeben:

- Welche Lernziele können mit der Aufgabe verfolgt werden?

- Wie wird dabei auf das mehrsprachige Repertoire der Lernenden zurückgegriffen?

- Wie findet ihr die Aufgabe?

\section{Begründung der Vorgehensweise}

Aufgrund der wahrscheinlich fehlenden Erfahrung aus der eigenen Schulzeit, die auf älteren Lehrplänen basierte, ist anzunehmen, dass die Studierenden nicht mit auf mehrsprachigkeitsdidaktischen Ansätzen basierenden und schon gar nicht mit plurilingualen Unterrichtsmaterialien / Aktivitäten vertraut sind. Durch die Aktivitäten an den Stationentischen lernen sie nicht nur verschiedene Übungen kennen, sondern können sie auch selbst ausprobieren. Die authentische Lernerfahrung und die daran anschließende Reflexion sollen dazu beitragen, dass die Studierenden motivierter, sicherer und kompetenter werden, wenn es darum geht, selbst als Lehrperson ähnliche Materialien im Unterricht einzusetzen.

\section{Bewertung}

Die Ausführlichkeit und Qualität der Vorbereitung der ersten Aufgabe (Lesen der Ausschnitte aus dem Lehrplan und Beantwortung der Fragen) wird bewertet. Dabei wird Augenmerk darauf gelegt, ob die Studierenden fähig sind, die Vorgaben des Lehrplans in einen weiteren theoretischen und auch gesellschaftlichen Rahmen zu setzen und mit den bisher in diesem Kurs behandelten Informationen in Verbindung zu setzen. Beim zweiten Teil der Lerneinheit wird kontrolliert, ob sich die Studierenden aktiv beim Ausprobieren und Diskutieren der Unterrichtsmaterialien beteiligen. Außerdem wird beurteilt, ob die Studierenden begründete Urteile in Bezug auf die Einsetzbarkeit der Materialien im Deutschunterricht bilden können. Die (erfolgreiche) Bewältigung der Aufgaben bzw. die plurilinguale Produktion im Zusammenhang mit der Durchführung der Aufgaben wird hier aber nicht beurteilt.

\section{Kursevaluation und Weiterentwicklung}

Das vorliegende Konzept für die Themeneinheit wurde im Herbst 2019 pilotiert. Leider konnte aus zeitlichen Gründen nicht das gesamte Programm umgesetzt werden, sondern einige Aufgaben wurden gekürzt. Alle Themen konnten aber, wenn auch nicht immer so ausführlich wie hier dargestellt, behandelt werden. Der Kontaktunterricht von sechs Stunden ist für diesen Stoff sehr knapp bemessen und reicht nur aus, wenn 
die Studierenden die Hausaufgaben gründlich machen und in den Stunden direkt darauf aufgebaut werden kann. Es wäre also sinnvoll, etwas mehr Kontaktunterricht für diese Themeneinheit zu reservieren, wenn das möglich ist. $\mathrm{Zu}$ sehen bleibt, ob der im Frühling $2020 \mathrm{im}$ Zuge der „Coronakrise“ notwendig gewordene Fernunterricht auch in Zukunft fortgesetzt wird. Große Teile der hier vorgestellten Unterrichtseinheiten können mit nur wenigen Adaptionen auch online durchgeführt werden. Das gesamte Material und viele Aufgaben wurden auch schon in der Pilotphase auf der Kursplattform Moodle zur Verfügung gestellt bzw. dort durchgeführt. Vorlesungen, Präsentationen und (Gruppen-)Diskussionen können in geeigneten Applikationen (Microsoft Teams oder Zoom) gehalten werden. Größerer Adaptionen bedarf es beim Kennenlernen und Ausprobieren der Aufgaben / Aktivitäten in der Lerneinheit 3. Die Studierenden müssten sich zuerst selbstständig mit den Materialien auseinandersetzen und in einem Videotreffen könnten dann die Erfahrungen im Plenum oder in Kleingruppen diskutiert werden.

Aufgrund des von den Studierenden erhaltenen Feedbacks und durch die von der Kursleiterin selbst durchgeführte Evaluation lassen sich folgende Punkte festhalten:

Die Inhalte der Themeneinheit erwiesen sich als sehr relevant und aktuell. Ein Teil der Studierenden absolvierte parallel zu diesem Kurs die Fachlehrerausbildung bzw. studierte im Hauptfach Klassenlehrerausbildung. Gerade von diesen Studierenden wurde in den Diskussionen immer wieder angesprochen, dass auch im Rahmen dieser Studien die Neuerungen in den Rahmenlehrplänen und die Konsequenzen, die sich daraus für den Unterricht ergeben, diskutiert wurden. Sie empfanden es als sehr positiv, dass in dieser Themeneinheit konkret auf die Rolle der Mehrsprachigkeit im Fremdsprachenunterricht eingegangen wurde.

Es zeigte sich, dass die eigenen Auffassungen der Kursteilnehmenden von Mehrsprachigkeit - wie auch in der schon erwähnten Umfrage (Grasz 2020) - teilweise älteren Definitionen entsprachen. Außerdem waren Erfahrungen mit auf mehrsprachigkeitsdidaktischen Ansätzen beruhenden bzw. plurilingualen Aktivitäten nur beschränkt vorhanden, obwohl alle Studierenden über ein breites mehrsprachiges Repertoire verfügten.

Die Verbindung von Theorie und Praxis, die im Kurs „Sprachdidaktik“ ein wichtiger Bestandteil ist, hat sich insgesamt als gelungen erwiesen. In Herbst 2019 war es leider nicht möglich, die Themeneinheit „Mehrsprachigkeit und DaF“ konkret in die Unterrichtspraxis umzusetzen, da das Thema erst in den letzten Stunden vor der Weihnachtspause behandelt wurde und keine Zeit mehr für Schulbesuche blieb. Alle Kursteilnehmer/innen haben aber Sprachduschen in Grundschulen organisiert und fanden die Schulbesuche sehr gewinnbringend. Aufgrund dieser Erfahrung ist es unbedingt wünschenswert, auch das Thema Mehrsprachigkeit im Rahmen von Schulbesuchen ausprobieren zu lassen. Wie oben schon erwähnt wurde, ist geplant, in Zukunft den Anwendungsteil des Kurses „Sprachdidaktik“ zu erweitern. Durch eine Studienplanreform ist es möglich, ab dem Studienjahr 2020/2021 gemeinsam mit dem Kurs einen 


\section{PlurioDeutsch}

Workshop anzubieten. Im Rahmen des Workshops bleibt mehr Zeit, die im Kurs behandelten Inhalte in die Praxis umzusetzen. Geplant ist dabei, dass die Studierenden eigene Unterrichtsstunden auch zum Thema Mehrsprachigkeit vorbereiten und in Partnerschulen halten - sofern Schulbesuche wieder möglich sind.

\section{Literatur}

Bertrand, Yves \& Christ, Herbert (1990): Vorschläge für einen erweiterten Fremdsprachenunterricht. Neusprachliche Mitteilungen 43: 4, 208-213.

Bloomfield, Leonard (1933): Language. New York: Holt, Rinehart and Winston.

Burwitz-Melzer, Eva; Mehlhorn, Grit; Riemer, Claudia; Bausch, Karl-Richard \& Krumm, Hans-Jürgen (Hrsg.) (2016): Handbuch Fremdsprachenunterricht. 6., völlig überarbeitete u. erw. Aufl. Tübingen: A. Francke.

Busch, Brigitta (2017): Mehrsprachigkeit. 2. Aufl. Wien: Facultas.

[CEF-Companion Volume] Council of Europe (2018): Common European Framework of Reference for Languages: Learning, Teaching, Assessment. Companion Volume with New Descriptors. Strasbourg: Council of Europe. https://rm.coe.int/cefr-companionvolume-with-new-descriptors-2018/ 1680787989, (19.12.2019).

[GeR] Europarat (2001): Gemeinsamer europäischer Referenzrahmen für Sprachen: Lernen, lehren, beurteilen. Berlin u.a.: Langenscheidt.

[GeR-Begleitband] Council of Europe (2020): Gemeinsamer europäischer Referenzrahmen für Sprachen Begleitband. Lernen, lehren, beurteilen; Begleitband mit Deskriptoren. Stuttgart: Ernst Klett Sprachen.

Grasz Sabine (2020): Die Rolle von Mehrsprachigkeit beim Lehren und Lernen von Fremdsprachen aus der Sicht zukünftiger SprachenlehrerInnen in Finnland. Zeitschrift für Interkulturellen Fremdsprachenunterricht 25: 1, 483-505.

https://tujournals.ulb.tu-darmstadt.de/index.php/zif/article/view/1042.

Grosjean, François (1982): Life with Two Languages. An Introduction to Bilingualism. Cambridge, Massachusetts, and London: Harvard University Press.

Hallet, Wolfgang \& Königs, Frank G. (2019): Mehrsprachigkeit und vernetzendes Sprachlernen. In: Hallet, Wolfgang \& Königs, Frank G. (Hrsg.): Handbuch Fremdsprachendidaktik. 3. Aufl. Seelze: Klett / Kallmeyer, 302-307.

Haugen, Einar Ingvald (1969): The Norwegian language in America: a study in bilingual behavior. Bloomington: Indiana University Press.

Krumm, Hans-Jürgen \& Reich, Hans H. (2016): Ansätze zum Mehrsprachigkeitsunterricht. In: Burwitz-Melzer et al. (Hrsg.): 230-234.

Kursiša, Anta \& Richter-Vapaatalo, Ulrike (2018) (Hrsg.): Mehr als Deutsch! Mehrsprachiges Lehrmaterial für den Deutschunterricht. Helsinki: Goethe-Institut Finnland. https://www.goethe.de/ins/fi/de/spr/unt/kum/dfj/eks.html.

[LOP 2019] Opetushallitus (Zentralamt für Unterrichtswesen) (2015): Lukion opetussuunnitelman perusteet [Lehrplan für die gymnasiale Oberstufe] www.oph.fi/sites/ default/files/documents/lukion_opetussuunnitelman_perusteet_2019.pdf (19.12.2019). 
Marx, Nicole (2016): Lernen von zweiten und weiteren Fremdsprachen im Sekundarschulalter. In: Burwitz-Melzer et al. (Hrsg.): 295-300.

Marx, Nicole \& Hufeisen, Britta (2010): Mehrsprachigkeitskonzepte. In: Krumm, HansJürgen; Fandrych, Christian; Hufeisen, Britta \& Riemer, Claudia (Hrsg.): Deutsch als Fremd- und Zweitsprache. Ein internationales Handbuch. 1. Halbband. Berlin: De Gruyter Mouton, 826-832.

Meißner, Franz-Joseph (2016): Interkomprehension. In: Burwitz-Melzer et al. (Hrsg.): 234-239.

[NCCBE 2016] Finnish National Board of Education (2016): National Core Curriculum for Basic Education 2014. Helsinki.

[POP 2014] Opetushallitus (Zentralamt für Unterrichtswesen) (2014): Perusopetuksen opetussuunnitelman perusteet [Lehrplan für die gemeinsame Gesamtschule]. https://eperusteet.opintopolku.fi/\#/fi/perusopetus/419550/tiedot (8.1.2020). 


\section{PlurioDeutsch}

\section{Anhang 1}

\section{Exemplarische Definitionen zu Mehrsprachigkeit aus verschiedenen Zeiten:}

In the cases where this perfect foreign-language learning is not accompanied by loss of the native language, it results in bilingualism, [the] native-like control of two languages. (Bloomfield 1933, zit. nach Grosjean 1982, S. 232)

Bilingualism ... may be of all degrees of accomplishment, but it is understood here to begin at the point where the speaker of one language can produce complete, meaningful utterances in the other language. From here it may proceed through all possible gradations up to the kind of skill that enables a person to pass as a native in more than one linguistic environment. (Haugen 1969, zit. nach Grosjean 1982, S. 232)

Als mehrsprachig darf schon der bezeichnet werden, der auf der Basis der Kenntnis seiner Muttersprache eingeschränkte Kenntnisse in wenigstens zwei weiteren Sprachen entweder in gleichen oder in unterschiedlichen Diskursbereichen hat (um z.B. soziale Kontakte in gesprochener oder geschriebener Sprache aufnehmen oder Texte lesen oder Fachgespräche führen zu können). (Betrand \& Christ 1990, S. 208)

,Mehrsprachigkeit' unterscheidet sich von ,Vielsprachigkeit', also der Kenntnis einer Anzahl von Sprachen oder der Koexistenz verschiedenere Sprachen in einer bestimmten Gesellschaft. Vielsprachigkeit kann man erreichen, indem man einfach das Sprachenangebot in einer Schule oder in einem Bildungssystem vielfältig gestaltet und indem man Schüler dazu anhält, mehr als eine Sprache zu lernen, oder indem man die dominante Stellung des Englischen in internationaler Kommunikation beschränkt. Mehrsprachigkeit jedoch betont die Tatsache, dass sich die Spracherfahrung eines Menschen in seinen kulturellen Kontexten erweitert, von der Sprache im Elternhaus über die Sprache der ganzen Gesellschaft bis zu den Sprachen anderer Völker (die er entweder in der Schule oder auf der Universität lernt oder durch direkte Erfahrung erwirbt). Diese Sprachen und Kulturen werden aber nicht in strikt voneinander getrennten mentalen Bereichen gespeichert, sondern bilden vielmehr gemeinsam eine kommunikative Kompetenz, zu der alle Sprachkenntnisse und Spracherfahrungen beitragen und in der die Sprachen miteinander in Beziehung stehen und interagieren. In verschiedenen Situationen können Menschen flexibel auf verschiedene Teile dieser Kompetenz zurückgreifen, um eine effektive Kommunikation mit einem bestimmten Gesprächspartner zu erreichen. Zum Beispiel können Gesprächspartner von einer Sprache oder einem Dialekt zu einer oder einem anderen wechseln und dadurch alle Möglichkeiten der jeweiligen Sprache oder Varietät ausschöpfen, indem sie sich z.B. in einer Sprache ausdrücken und den Partner in der anderen verstehen. Man kann auch auf die Kenntnis mehrerer Sprachen zurückgreifen, um den Sinn eines geschriebenen oder gesprochenen Textes zu verstehen, der in einer eigentlich unbekannten Sprache verfasst wurde; dabei erkennt man zum Beispiel Wörter aus einem Vorrat an Internationalismen, die hier nur in neuer Gestalt auftreten. Jemand mit - wenn vielleicht auch nur geringen - Sprachkenntnissen kann diese benutzen, um anderen, die über gar keine verfügen, bei der Kommunikation zu helfen, indem er zwischen den Gesprächspartnern ohne gemeinsame Sprache sprachmittelnd aktiv wird. Auch wenn kein Sprachmittler zur Verfügung steht, können solche Menschen trotzdem bis zu einem gewissen Grad kommunizieren, indem sie ihren ganzen Vorrat an linguistischem Wissen ins Spiel bringen und mit alternativen Formen des Ausdrucks in verschiedenen Sprachen oder Dialekten experimentieren und dabei paralinguistische Mittel nutzen (Mimik, Gestik, Gesichtsausdruck usw.) und ihre Sprache radikal vereinfachen. (GER 2001, S. 17) 
Mehrsprachigkeit - der Begriff ist nicht unproblematisch und wird innerhalb der angewandten Sprachwissenschaft zunehmend kritisch hinterfragt. Lange Zeit wurde die Beschäftigung mit Mehrsprachigkeit vor allem ausgehend vom englischen Sprachraum unter dem Begriff Bilingualismus-Forschung zusammengefasst, womit die Zweisprachigkeit als Sonderfall von der als Normalfall betrachteten Einsprachigkeit abgegrenzt wurde. Manche benützten in der Folge auch den Begriff Trilingualismus, um dem Umstand Rechnung zu tragen, dass oft mehr als zwei Sprachen im Spiel sind. Um die Reihenfolge des Spracherwerbs oder Hierarchisierung im individuellen sprachlichen Repertoire zu bezeichnen, hat man Begriffe wie Erstsprache, Zweitsprache, L1, L2, L3, Ln eingeführt. Das impliziert die Annahme, dass Sprachen klar voneinander abgrenzbar und somit zählbar sind.

Seit einigen Jahren wird in der angewandten Sprachwissenschaft darüber diskutiert, wie aus der Perspektive des Fachs Sprache gefasst und verstanden werden kann. Hinterfragt wird dabei vor allem die Vorstellung von Sprachen als voneinander klar abgegrenzten Entitäten, also die Annahme, dass Sprachen wie etwa Deutsch, Englisch, Russisch im Gebrauch trennscharf unterschieden werden können [...]. (Busch 2017, S. 9)

\section{Quellen:}

Bertrand, Yves \& Christ, Herbert (1990): Vorschläge für einen erweiterten Fremdsprachenunterricht. Neusprachliche Mitteilungen 43: 4, 208-213.

Bloomfield, Leonard (1933): Language. New York: Holt, Rinehart and Winston.

Busch, Brigitta (2017): Mehrsprachigkeit. 2. Auflage. Wien: Facultas Verlag.

Grosjean, François (1982): Life with Two Languages. An Introduction to Bilingualism. Cambridge, Massachusetts, and London: Harvard University Press.

Haugen, Einar Ingvald (1969): The Norwegian language in America: a study in bilingual behavior. Bloomington: Indiana University Press.

Europarat (2001): Gemeinsamer europäischer Referenzrahmen für Sprachen: lernen, lehren, beurteilen. Berlin et al.: Langenscheidt. 


\section{PlurioDeutsch}

\section{Anhang 2}

National Core Curriculum for Basic Education 2014/NCCBE 2014 (erschienen 2016):

The development of language proficiency begins in early childhood and continues as a lifelong process. Plurilingual competence develops at home, at school, and during leisure time. It comprises competences of different levels in mother tongues, other tongues, and their dialects. The basic principal of language instruction at school is using the language in different situations. It strengthens the pupils' language awareness and parallel use of different languages as well as the development of multiliteracy. The pupils learn to make observations on texts and interaction practices in different languages, to use the concepts of language knowledge in interpreting texts, and to utilize diverse ways of language learning. The pupils use their skills in different languages as support for all learning in different subjects. (S. 132-133)

The objective is to guide the pupils to appreciate different languages and cultures and to promote bilingualism and plurilingualism, thus reinforcing the pupils' linguistic awareness and metalinguistic skills. School work may include multilingual teaching situations where the teachers and pupils use all languages they know. (S. 90)

The instruction of languages is a part of language education and introduction to language awareness. [...] The instruction also connects and makes use of different languages [...]. (S. 135-136)

The pupil is able to make observations on the differences and similarities related to structures, vocabulary, and other features of the language he or she studies and his or her mother tongue or another language he or she knows. (S. 243)

Linguistic concepts that help the pupils in making comparisons between languages and studying the target language are utilised. (S. 381)

\section{Quelle:}

[NCCBE 2016] Finnish National Board of Education (2016): National Core Curriculum for Basic Education 2014. Helsinki. 